\title{
CONFORMAL VARIATION OF A HYPERSURFACE OF CONSTANT MEAN CURVATURE IN AN EINSTEIN SPACE
}

\author{
GEOFFREY HOWARD SMITH \\ (Received 6 May 1981; revised 8 October 1981) \\ Communicated by K. Mackenzie
}

\begin{abstract}
Let a compact orientable manifold be immersed as a hypersurface of constant mean curvature in an Einstein space. It is shown that the immersion is totally umbilical if and only if there exists a conformal variation of the immersion whose variation vector is nowhere tangential to the hypersurface.
\end{abstract}

1980 Mathematics subject classification (Amer. Math. Soc.): 53 C 42, 53 C 40.

\section{Introduction}

A theorem of Liebmann (1900) states that the only ovaloids of constant mean curvature in Euclidean 3-space are spheres. The proof uses an integral formula of Minkowski and so to generalise Liebmann's theorem one can begin by developing integral formulas for hypersurfaces in a Riemannian space. To do this one needs a replacement for the position vector which plays an important part in the Euclidean case. One method of doing this is to assume the existence of a vector field on the enveloping space with certain specified properties. For example we have the following theorem (Katsurada (1964)).

THEOREM. Let $R^{m+1}$ be an Einstein space, $V^{m}$ a closed orientable hypersurface with $H_{1}=$ constant in $R^{m+1}$; we suppose that there exists a continuous one-parameter group $G$ of conformal transformations of $R^{m+1}$ such that the scalar product $p=n^{i} \xi_{i}$ of the normal vector $n$ of $V^{m}$ and the vector $\xi$ belonging to $G$ does not change the sign (and is not $\equiv 0$ ) on $V^{m}$. Then every point of $V^{m}$ is umbilic.

(c) Copyright Australian Mathematical Society 1982 
A problem arises when immersed hypersurfaces are considered since it is quite easy to construct totally umbilical immersions in an Einstein space for which no conformal vector field satisfies the hypotheses of the theorem. Hence Katsurada's theorem does not provide necessary conditions.

EXAMPLE. Let $M_{b}$ be the subset of $E^{3}$ (Euclidean 3-space) defined by

$$
M_{b}=\left\{(x, y, z) \in E^{3} \mid-1 \leqslant x \leqslant 1,-2<y, z<2\right\} .
$$

The subset $\left\{(x, y, z) \in M_{b} \mid x^{2}+y^{2}+z^{2}=1\right\}$ is the image of the two sphere $S^{2}$ under some map $f: S^{2} \rightarrow M_{b}$. Now form a flat Einstein manifold $M$ from $M_{b}$ by taking $M$ to be the quotient space under the identification $(-1, y, z) \sim(1, y, z)$ for all $y, z \in(-2,2)$. Denote the projection by $\pi: M_{b} \rightarrow M$. The map $\pi \circ f$ : $S^{2} \rightarrow M$ is a totally umbilical immersion of $S^{2}$ in the Einstein space $M$.

If $V^{\prime}$ is a vector field on an open set $U \subset E^{3}$ containing $M_{b}$ then we can define a vector field $V$ on $M_{b}$ by letting $V=V^{\prime} \mid M_{b}$. In order for $\pi_{*}(V)$ to be a vector field on $M$ it is necessary and sufficient that $v^{\alpha}(-1, y, z)=v^{\alpha}(1, y, z)$, where $v^{\alpha}$, $\alpha=1,2,3$, are the components of $V$. This condition results in the group of conformal transformations of $M$ being only 4 dimensional, rather than 10 dimensional as in the case of $E^{3}$. In fact, a vector field on $M$ is conformal if and only if it is of the form

$$
\pi_{*}\left(a \frac{\partial}{\partial x}+b \frac{\partial}{\partial y}+c \frac{\partial}{\partial z}+d\left(y \frac{\partial}{\partial z}-z \frac{\partial}{\partial y}\right)\right),
$$

where $a, b, c$ and $d$ are arbitrary constants.

It is now easy to check that if $N$ is a unit normal vector field over $\pi \circ f$ (we explain this concept below) and if $W$ is a conformal vector field on $M$ then $\langle N, W\rangle$ is either identically zero on $(\pi \circ f)\left(S^{2}\right)$ or $\langle N, W\rangle$ changes sign on $(\pi \circ f)\left(S^{2}\right)$. Here $\langle$,$\rangle denotes the natural inner product on M$.

Katsurada's theorem is inapplicable to this simple example. To derive an applicable theorem we need a replacement for the requirement involving the conformal vector field. We now show how to do this.

\section{Conformal variations}

In what follows we use the notation of Kobayashi and Nomizu (1963) pages 313-314, unless stated otherwise. Let $f: M \rightarrow M^{\prime}$ be an immersion of a manifold $M$ as a hypersurface of a Riemannian manifold $M^{\prime}$. We give $M$ the induced metric. A vector field over the map $f$ is a smooth map

$$
W: M \rightarrow T\left(M^{\prime}\right)
$$


such that

$$
W(p) \in T_{f(p)}\left(M^{\prime}\right) \text { for all } p \in M \text {. }
$$

Since $f$ need not be injective it is possible to have distinct points $p, q \in M$ with $f(p)=f(q)$ but $W(p)=W(q)$, so that $W$ need not be the restriction to $f(M)$ of any vector field on $M^{\prime}$.

Let $W$ be a vector field over $f$. For each $p \in M$ we write $W^{\top}(p)$ (resp. $\left.W^{\perp}(p)\right)$ for the component of $W(p)$ tangential (resp. normal) to $f(M)$. For each $p \in M$ there exist open neighbourhoods $V$ of $p$ and $U$ of $f(p)$ with $f(V) \subset U$ and a smooth vector field $\mathbf{W}$ on $U$ such that $\mathbf{W}(f(q))=W(q)$ for all $q \in V$. Then

$$
\left(L_{\mathbf{W}} g\right)(X, Y)=\left\langle D_{X}^{\prime} \mathbf{W}, Y\right\rangle+\left\langle D_{Y}^{\prime} \mathbf{W}, X\right\rangle \text {, }
$$

where $X$ and $Y$ are tangent to $f(M), D^{\prime}$ denotes covariant differentiation on $M^{\prime}$ and $g$ or $\langle$,$\rangle denotes (according to convenience) the metric on M^{\prime}$.

Let $N: M \rightarrow T\left(M^{\prime}\right)$ be a vector field over $f$ with $N(p)=N^{\perp}(p)$ and $\langle N(p), N(p)\rangle=1$ for all $p \in M$. The evaluation of tensor quantities at any particular point is a purely local matter, and since an immersion is locally an imbedding it simplifies matters to assume that $f$ actually is an imbedding and to identify $M$ and $f(M)$. We shall do this unless (as in the example above) it is necessary to distinguish between an immersion and an imbedding. The following results hold (Kobayashi and Nomizu (1969) page 15):

$$
\begin{array}{ll}
D_{X}^{\prime} N=-A_{N} X & \text { (Weingarten's formula), } \\
D_{X}^{\prime} Y=D_{X} Y+\left\langle A_{N} X, Y\right\rangle N & \text { (Gauss' formula), }
\end{array}
$$

where $D$ denotes covariant differentiation on $M, A_{N}$ is the second fundamental tensor and $X, Y$ are tangent vector fields on $M$. Applying these results to (1) yields

$$
\left(L_{\mathbf{w}} g\right)(X, Y)=\left\langle D_{X} W^{\top}, Y\right\rangle+\left\langle D_{Y} W^{\top}, X\right\rangle-2\left\langle A_{N} X, Y\right\rangle\langle N, W\rangle .
$$

The significance of this equation is that the right hand side depends only on $W$ and not the particular extension $\mathbf{W}$ of $W$. Hence we can unambiguously write $\left(L_{W} g\right)(X, Y)$ for $\left(L_{\mathrm{w}} g\right)(X, Y)$ if $X$ and $Y$ are tangent to $M$.

We say that a vector field $W$ over $f$ is a conformal variation of $f(M)$ if $\left(L_{W} g\right)\left(f_{*} X, f_{*} Y\right)=2 \phi\left\langle f_{*} X, f_{*} Y\right\rangle$ for some scalar $\phi$ and any vector fields $X$ and $Y$ on $M$.

Our main result is the following theorem.

THEOREM. Let $f: M \rightarrow M^{\prime}$ be an immersion of a compact orientable manifold as a hypersurface of an $n+1$ dimensional Einstein space $M^{\prime}$, and suppose that the mean curvature of $f(M)$ in $M^{\prime}$ is constant. A necessary and sufficient condition that the immersion be totally umbilical is that there exist a conformal variation $W$ of $f(M)$ which is nowhere tangent to $f(M)$. 
Proof. Necessity is easily disposed of; put $W=N$. Then by equation (2)

$$
\left(L_{N} g\right)(X, Y)=-2\left\langle A_{N} X, Y\right\rangle \text {. }
$$

Since the immersion is totally umbilical we have $A_{N} X=-\phi X$ for some scalar $\phi$. Hence

$$
\left(L_{N} g\right)(X, Y)=2 \phi\langle X, Y\rangle
$$

so that $N$ is a conformal variation of $f(M)$.

\section{Two integral formulas}

Let $\left\{e_{1}, \ldots, e_{n}, N\right\}$ be an orthonormal moving frame on an open set of $M$. By (2) we have

$$
\sum_{\alpha=1}^{n}\left(L_{W} g\right)\left(e_{\alpha}, e_{\alpha}\right)=2 \operatorname{div} W^{\top}-2 n H_{1}\langle N, W\rangle
$$

where

$$
H_{1}=\frac{1}{n} \sum_{\alpha=1}^{n}\left\langle A_{N} e_{\alpha}, e_{\alpha}\right\rangle
$$

is the first mean curvature of $M$ in $M^{\prime}$. If we assume that the variation $W$ is conformal and that $M$ is compact and orientable then Stokes' theorem yields

$$
\int_{M}\left[H_{1}\langle N, W\rangle+\phi\right] d A=0 .
$$

We remark that if the distinction between $M$ and $f(M)$ is made, then $W^{\top}$ in (3) by abuse of notation really denotes the unique vector field $Z$ on $M$ such that $f_{*} Z=W^{\top}$. It is to this vector field $Z$ that Stokes' theorem is applied to yield (4). Similar considerations apply to our next integral formula.

On some open set $V$ of $M$ choose an orthonormal moving frame $\left\{e_{1}, \ldots, e_{n}, N\right\}$ such that $e_{\alpha}$ is an eigenvector with eigenvalue $\lambda_{\alpha}(\alpha=1, \ldots, n)$ for the self-adjoint operator $A_{N}$. We define a vector field $P \in T(V)$ by letting

$$
\langle P, X\rangle=\left\langle D_{X}^{\prime} N, W\right\rangle, \quad X \in T(V) .
$$

Then

$$
\operatorname{div} P=\sum_{\alpha=1}^{n}\left\langle D_{e_{\alpha}} P, e_{\alpha}\right\rangle=\sum\left[e_{\alpha}\left(\left\langle P, e_{\alpha}\right\rangle\right)-\left\langle P, D_{e_{\alpha}} e_{\alpha}\right\rangle\right]
$$

so that by Weingarten's formula

$$
\operatorname{div} P=\sum\left[e_{\alpha}\left(\left\langle P, e_{\alpha}\right\rangle\right)+\left\langle D_{e_{\alpha}} e_{\alpha}, A_{N} W^{\top}\right\rangle\right]
$$


To evaluate the first term on the right hand side of (5) we note that

$$
\begin{aligned}
e_{\alpha}\left(\left\langle P, e_{\alpha}\right\rangle\right) & =e_{\alpha}\left(\left\langle D_{e_{\alpha}}^{\prime} N, W\right\rangle\right) \\
& =\left\langle D_{e_{\alpha}^{\prime}}^{\prime} D_{e_{\alpha}^{\prime}}^{\prime} N, W\right\rangle+\left\langle D_{e_{\alpha}}^{\prime} N, D_{e_{\alpha}}^{\prime} W\right\rangle,
\end{aligned}
$$

and using the Gauss and Weingarten formulas we find

$$
e_{\alpha}\left(\left\langle P, e_{\alpha}\right\rangle\right)=-\left\langle D_{e_{\alpha}}\left(\lambda_{\alpha} e_{\alpha}\right), W\right\rangle-\left\langle\lambda_{\alpha} e_{\alpha}, D_{e_{\alpha}} W^{\top}\right\rangle .
$$

From Codazzi's equation (Kobayashi and Nomizu (1969) page 25) we have

$$
\left\langle R^{\prime}\left(e_{\alpha}, W^{\tau}\right) e_{\alpha}, N\right\rangle=\left(D_{e_{\alpha}} B\right)\left(e_{\alpha}, W^{\top}\right)-\left(D_{W^{\top}} B\right)\left(e_{\alpha}, e_{\alpha}\right),
$$

where $B(X, Y)=\left\langle A_{N} X, Y\right\rangle$. Hence if $M^{\prime}$ is an Einstein space

$$
0=-\operatorname{Ric}^{\prime}\left(W^{\top}, N\right)
$$

so that

$$
\begin{aligned}
0=\sum\left[e_{\alpha}\left(B\left(e_{\alpha}, W^{\top}\right)\right)-\right. & B\left(D_{e_{\alpha}} e_{\alpha}, W^{\top}\right) \\
& \left.-B\left(e_{\alpha}, D_{e_{\alpha}} W^{\top}\right)+2 B\left(D_{W^{\top}} e_{\alpha}, e_{\alpha}\right)\right],
\end{aligned}
$$

where we have assumed that $n H_{1}=\Sigma B\left(e_{\alpha}, e_{\alpha}\right)$ is a constant. Hence from (7)

$$
\begin{aligned}
& 0=\Sigma\left[e_{\alpha}\left(\left\langle\lambda_{\alpha} e_{\alpha}, W^{\top}\right\rangle\right)-\left\langle A_{N} W^{\top}, D_{e_{\alpha}} e_{\alpha}\right\rangle-\lambda_{\alpha}\left\langle e_{\alpha}, D_{e_{\alpha}} W^{\top}\right\rangle\right], \\
& 0=\Sigma\left[\left\langle D_{e_{\alpha}}\left(\lambda_{\alpha} e_{\alpha}\right), W^{\top}\right\rangle-\left\langle A_{N} W^{\top}, D_{e_{\alpha}} e_{\alpha}\right\rangle\right] .
\end{aligned}
$$

Substitution of (6) into (8) yields

$$
\sum e_{\alpha}\left(\left\langle P, e_{\alpha}\right\rangle\right)=-\sum\left[\left\langle A_{N} W^{\top}, D_{e_{\alpha}} e_{\alpha}\right\rangle+\left\langle\lambda_{\alpha} e_{\alpha}, D_{e_{\alpha}} W^{\top}\right\rangle\right],
$$

so that from (5)

$$
\operatorname{div} P=-\sum \lambda_{\alpha}\left\langle e_{\alpha}, D_{e_{\alpha}} W^{\top}\right\rangle .
$$

On the other hand from (2)

$$
\begin{aligned}
\sum \lambda_{\alpha}\left(L_{W} g\right)\left(e_{\alpha}, e_{\alpha}\right) & =2 \sum\left[\lambda_{\alpha}\left\langle e_{\alpha}, D_{e_{\alpha}} W^{\top}\right\rangle-\lambda_{\alpha}\left\langle\lambda_{\alpha} e_{\alpha}, e_{\alpha}\right\rangle\langle N, W\rangle\right] \\
& =2 \sum\left[\lambda_{\alpha}\left\langle e_{\alpha}, D_{e_{\alpha}} W^{\top}\right\rangle-\lambda_{\alpha}^{2}\langle N, W\rangle\right] .
\end{aligned}
$$

Using

$$
\begin{aligned}
\sum \lambda_{\alpha}^{2} & =\left(\sum \lambda_{\alpha}\right)^{2}-2 \sum_{\alpha<\beta} \lambda_{\alpha} \lambda_{\beta} \\
& =n^{2} H_{1}^{2}-2\left(\begin{array}{l}
n \\
2
\end{array}\right) H_{2}
\end{aligned}
$$

gives us the result

$\sum \lambda_{\alpha}\left(L_{W} g\right)\left(e_{\alpha}, e_{\alpha}\right)=2 \sum\left[\lambda_{\alpha}\left\langle e_{\alpha}, D_{e_{\alpha}} W^{\tau}\right\rangle\right]-\left(n^{2} H_{1}^{2}-n(n-1) H_{2}\right)\langle N, W\rangle$. 
Substitution of this result into (9) gives

$$
\operatorname{div} P=-\left(n^{2} H^{2}-n(n-1) H_{2}\right)\langle N, W\rangle-\frac{1}{2} \sum \lambda_{\alpha}\left(L_{W} g\right)\left(e_{\alpha}, e_{\alpha}\right) .
$$

If the variation $W$ is conformal and $M$ is compact and orientable then

$$
\int_{M}\left[\left(n H_{1}^{2}-(n-1) H_{2}\right)\langle N, W\rangle+\phi H_{1}\right] d A=0 .
$$

Multiplication of equation (4) by $H_{1}$ (= constant) and subtraction from (10) yields

$$
\int_{M}(n-1)\left(H_{1}^{2}-H_{2}\right)\langle N, W\rangle d A=0 .
$$

If $W$ is nowhere tangent to $M$ then $\langle N, W\rangle$ is of constant sign and $H_{1}^{2}-H_{2}=0$, from which we find $\lambda_{1}=\lambda_{2}=\cdots=\lambda_{n}$ and the immersion is totally umbilical. This completes the proof of the theorem.

\section{References}

Y. Katsurada (1964), 'On a certain property of closed hypersurfaces in an Einstein space'. Comment. Math. Helv. 38, 165-171.

S. Kobayashi and K. Nomizu (1963), Foundations of differential geometry, Volume 1 (Interscience, New York).

S. Kobayashi and K. Nomizu (1969), Foundations of differential geometry, Volume 2 (Interscience, New York).

H. Liebmann (1900), 'Über die Verbiegung der geschlossenen Flächen positiver Krümmung', Math. Ann. 53, 91-112.

The New South Wales Institute of Technology

P. O. Box 123

Broadway, New South Wales 2007

Australia 